\title{
EDITORIAL
}

\section{Long-acting inhaled bronchodilators in COPD: how many drugs do we need?}

\author{
P.M.A. Calverley
}

B ronchodilators remain the mainstay of chronic obstructive pulmonary disease (COPD) management and are recommended for the treatment of symptomatic patients at all stages of this disease [1]. For many years studies of these drugs focussed on changes in forced expiratory volume in one second (FEV1) or, occasionally, forced vital or slow vital capacity, believing that these robust and reproducible end-points were a legitimate surrogate for the changes in symptomatology, which were the real interest of patients and clinicians alike. By the 1990s, this comfortable assumption had been challenged when it became clear that individual improvement in exercise capacity and breathlessness were not closely related to changes in the magnitude of these spirometric indices [2]. This stimulated research into the determinants of exercise performance and dyspnoea in COPD, and why spirometry was of so little help in predicting symptomatic improvement. A range of mechanisms have been identified and have recently been reviewed [3]. Principal among these is the change in end-expiratory lung volume (EELV) during exercise in COPD patients. When healthy subjects exercise, EELV is reduced helping to keep the operating lung volumes on the steeper part of the pressurevolume relationship. COPD patients with tidal expiratory flow limitation must either allow EELV to rise [4], or try to overcome flow limitation at their initial lung volume an unsatisfactory strategy which limits exercise performance [5]. Most patients with more severe disease adopt for the former approach which allows them to exercise longer, but at the cost of increasing difficulty in breathing as EELV approaches total lung capacity [6].

Several studies have examined exercise performance in light of this improved mechanistic understanding. Studies with the shorter acting anticholinergic drug ipratropium confirmed that the degree of spirometric change was unrelated to any improvement in exercise performance [7], but that increases in inspiratory capacity during exercise did identify individuals who could exercise for longer. Further data with both tiotropium and salmeterol $[6,8]$ have shown that these bronchodilator drugs lower resting EELV which creates more "room" within the thorax to accommodate the volume change that exercise produces. At any time during exercise the degree of breathlessness is less after the bronchodilator drug, although at end-exercise when the inspiratory reserve volume approaches $500 \mathrm{~mL}$ a rapid increase in self-reported breathlessness occurs.

CORRESPONDENCE: P.M.A. Calverley, The University Hospital Aintree, Liverpool, L9 7AL, UK. Fax: 44 1515295888. E-mail: pmacal@liverpool.ac.uk
This reflects the substantial neuro-mechanical dissociation which occurs when EELV approaches total lung capacity. Data with salmeterol suggests that the main effect of this drug is to delay the time taken to reach this point [6], although other subtler mechanisms have been proposed [9].

Thus, changes in operating lung volume explain why bronchodilators make people less breathless and why small changes in mean FEV1, measured as an average change in groups of COPD patients, rather than individuals, can be accompanied by important clinical benefits. However, detailed mechanistic studies of this type are too complex to answer all our questions about how and when to use bronchodilator drugs, and in many circumstances we are still reliant on detecting small changes in spirometry to guide clinical practice. This is especially true when we determine the duration of action of drugs and whether combining drugs is better than using either one alone.

Although differences in the pharmacology of individual bronchodilators have been a topic of much discussion, the most useful practical development has been to prolong their duration of action. Despite differences in dissociation times for different muscarinic receptor subtypes, tiotropium improves exercise performance in COPD patients in a similar way to short-acting anticholinergic drugs $[7,8]$. However, tiotropium can be taken $q . d$. and produces improvements in spirometry for $\geqslant 24 \mathrm{~h}$, including the overnight period [10]. This sustained action maintains airway patency, which previously we have argued to be desirable in COPD [11]. This is the most likely reason for the superior clinical performance of this agent compared with its short-acting counterparts [12].

Although tiotropium is a very effective anticholinergic drug, it is unlikely that the current dosing regime is on the flat part of the dose-response relationship [13], with the dose of $18 \mu \mathrm{g}$ q.d. being chosen to represent a reasonable compromise between clinical efficacy and the risk of systemic absorption and toxicity. Indeed, combining anticholinergic and $\beta$-agonist in high doses can still produce significant improvements in lung function and lung volume irrespective of the presence of expiratory flow limitation [14]. This has been the basis of the widely used regular shortacting combination drugs in COPD [15]. Inevitably, clinicians would also like to know whether there is any benefit in combining long-acting inhaled drugs as well and in this issue of the European Respiratory Journal, a helpful study is reported which begins to address this complex problem [16].

In the present issue, vAN NOORD et al. [16] compared tiotropium q.d., the long-acting inhaled $\beta$-agonist formoterol b.i.d., and the 
combination of tiotropium q.d. and formoterol q.d. in a randomised placebo-controlled crossover trial in 71 patients with stable COPD (mean FEV1 37\% predicted). Patients took each treatment for 6 weeks at the end of which spirometry was recorded 17 times over the subsequent $24 \mathrm{~h}$ to identify early and more sustained changes in lung function after each drug. Regular peak flow recordings were made and rescue medication use was noted, but other measurements such as inspiratory capacity were not added to what was already a demanding protocol

Several points emerged clearly. The patients enrolled were very similar to those reported in previous studies of tiotropium [12, 17-19] and, as anticipated, there was a significant improvement in mean trough FEV1 of $\sim 100 \mathrm{~mL}$ after this drug. This is comparable with other studies and was significantly greater than that after formoterol (b.i.d.). There was further significant improvement in spirometry over the first $12 \mathrm{~h}$ after the two drugs were taken together. As noted previously [10], the diurnal variation in lung function persists despite inhaled tiotropium therapy and there was no evidence of any residual effect from the morning dose of formoterol over the second half of the day. Similar changes were seen in peak expiratory flow and drug combination was well tolerated. There was a greater reduction in the daytime use of "rescue" salbutamol during treatment with the combination compared with the individual components, but this was the only clinically important comparator included in this study.

The present data are interesting although largely limited to spirometric outcomes and represent an unusual dosing regime for clinical purposes. The crossover design of the study is a considerable strength, as is the detailed time course data after sustained dosing with these drugs. There is clear evidence of additional benefit when the drugs are combined and this is lost as the formoterol effect wears off. There is currently considerable interest in developing q.d. inhaled therapy that combines the undoubted benefits of tiotropium with an equally longacting $\beta$-agonist. Formoterol q.d. does not meet this requirement, but b.i.d. therapy would appear to be worth exploring in studies where relevant clinical end-points are evaluated.

The persistent increase in airflow obstruction that characterises chronic obstructive pulmonary disease is best addressed by improving lung function throughout the 24-h day and certainly during the period when patients are awake and most active. The new data from VAN NOORD et al. [16] suggests that, to achieve this goal, we can do more than we currently are. In this setting, modest improvements spirometrically often indicate larger functionally important changes. The challenge for the future will be to establish whether the clinical benefit produced by combining these drug classes merits the additional expense and potential inconvenience of adding another inhaler to an already complex treatment regime.

\section{REFERENCES}

1 Celli BR, MacNee W. Standards for the diagnosis and treatment of patients with COPD: a summary of the ATS/ ERS position paper. Eur Respir J 2004; 23: 932-946.

2 Hay JG, Stone P, Carter J, et al. Bronchodilator reversibility, exercise performance and breathlessness in stable chronic obstructive pulmonary disease. Eur Respir J 1992; 5: 659-664.

3 Calverley PM. Breathlessness during exercise in COPD: how do the drugs work?. Thorax 2004; 59: 455-457.

4 O'Donnell DE, Revill SM, Webb KA. Dynamic hyperinflation and exercise intolerance in chronic obstructive pulmonary disease. Am J Respir Crit Care Med 2001; 164: 770-777.

5 Aliverti A, Stevenson N, Dellaca RL, Lo MA, Pedotti A, Calverley PM. Regional chest wall volumes during exercise in chronic obstructive pulmonary disease. Thorax 2004; 59: 210-216.

6 O'Donnell DE, Voduc N, Fitzpatrick M, Webb KA. Effect of salmeterol on the ventilatory response to exercise in chronic obstructive pulmonary disease. Eur Respir J 2004; 24: 86-94.

7 O'Donnell DE, Lam M, Webb KA. Spirometric correlates of improvement in exercise performance after anticholinergic therapy in chronic obstructive pulmonary disease. Am J Respir Crit Care Med 1999; 160: 542-549.

8 O'Donnell DE, Fluge T, Gerken F, et al. Effects of tiotropium on lung hyperinflation, dyspnoea and exercise tolerance in COPD. Eur Respir J 2004; 23: 832-840.

9 Man WD-C, Mustfa N, Nikoletou D, et al. The effect of salmeterol on respiratory muscle activity during exercise in poorly reversible COPD. Thorax 2004; 59: 471-476.

10 Calverley PM, Lee A, Towse L, van Noord J, Witek TJ, Kelsen S. Effect of tiotropium bromide on circadian variation in airflow limitation in chronic obstructive pulmonary disease. Thorax 2003; 58: 855-860.

11 Rennard SI, Calverley P. Rescue! Therapy and the paradox of the Barcalounger. Eur Respir J 2003; 21: 916-917.

12 Vincken W, Van Noord JA, Greefhorst AP, et al. Improved health outcomes in patients with COPD during $1 \mathrm{yr}$ 's treatment with tiotropium. Eur Respir J 2002; 19: 209-216.

13 Barnes PJ. The pharmacological properties of tiotropium. Chest 2000; 117: Suppl. 2, 63S-66S.

14 Hadcroft J, Calverley PM. Alternative methods for assessing bronchodilator reversibility in chronic obstructive pulmonary disease. Thorax 2001; 56: 713-720.

15 In chronic obstructive pulmonary disease, a combination of ipratropium and albuterol is more effective than either agent alone. An 85-day multicenter trial. COMBIVENT Inhalation Aerosol Study Group. Chest 1994; 105: 1411-1419.

16 van Noord JA, Aumann J-L, Jansseens E, et al. Comparison of tiotropium once daily, formoterol twice daily and both combined once daily in patients with COPD. Eur Respir J 2005; 26: 214-222.

17 Casaburi R, Mahler DA, Jones PW, et al. A long-term evaluation of once-daily inhaled tiotropium in chronic obstructive pulmonary disease. Eur Respir J 2002; 19: 217-224.

18 Celli B, ZuWallack R, Wang S, Kesten S. Improvement in resting inspiratory capacity and hyperinflation with tiotropium in COPD patients with increased static lung volumes. Chest 2003; 124: 1743-1748.

19 McNicholas WT, Calverley PM, Lee A, Edwards JC. Longacting inhaled anticholinergic therapy improves sleeping oxygen saturation in COPD. Eur Respir J 2004; 23: 825-831. 